\title{
Effects of plasma shape on laser blow-off injected impurity transport in TCV
}

\author{
E. Scavino, J.S. Bakos ${ }^{1}$, R. Dux ${ }^{2}$, H. Weisen and TCV Team \\ Centre de Recherches en Physique des Plasmas, \\ Association EURATOM - Confédération Suisse, \\ Ecole Polytechnique Fédérale de Lausanne \\ CH-1015 Lausanne, Switzerland \\ ${ }^{1}$ KFKI Research Institute for Particle and Nuclear Physics, Budapest, H-1525 Hungary \\ ${ }^{2}$ Max-Planck-Institut für Plasmaphysik, Euratom Association, Garching, Germany \\ E-mail address of E. Scavino: scavino@ crppsun3.epfl.ch \\ PACS: 52.25.Vy
}

\begin{abstract}
The extreme shaping capabilities of the TCV tokamak have been used to investigate the effect of the plasma geometry on the confinement of non recycling trace impurities injected by means of the laser blow-off technique. The progression of the injected Silicon in the core of TCV Ohmic limiter plasmas was followed by the 200-channel soft x-ray photodiode array with good spatial and temporal resolution. The results show that the plasma triangularity and elongation play an important role in the impurity confinement time $\tau_{i m p}$. Remarkably, the increase of elongation from $\kappa=1.6$ to $\kappa=2.3$ produces a threefold reduction of $\tau_{\text {imp }}$ while the electron energy confinement time $\tau_{E e}$ remains almost constant. $\tau_{i m p}$ is fairly constant in the triangularity scan for $\delta>0.2$ while there is a marked increase for lower values, leading to $\tau_{\text {imp }}>100 \mathrm{~ms}$ for negative triangularities. The increase of the toroidal magnetic field $B_{T}$ from $B_{T}=0.92 \mathrm{~T}$ to $B_{T}=1.47 \mathrm{~T}$ produces a decrease in the confinement time by almost of a factor-of-two. Simulation of the evolution of the line integrated soft x-ray signals, performed by the 1-D code STRAHL, provided both central and peripheral values of the transport coefficients together with estimates of the radial profiles. The simulations show that anomalous transport is dominant over neoclassical transport, except near the plasma centre. Interestingly, the convective velocity is positive (outwardly directed) in all limiter cases.
\end{abstract}

\section{Introduction}

Impurities determine radiation losses, contribute to fuel dilution and hence play an important role in fusion plasmas. In view of advanced, strongly shaped reactor designs, one important 
and frequently neglected aspect is the effect of plasma geometry on the confinement of fuel particles and impurities, specifically in relation with the energy confinement.

The main goal of the TCV tokamak is the study of the effect of different plasma shapes on confinement and stability[1]. Extensive investigations were carried out on the confinement of the energy in Ohmic discharges. The energy confinement time of the plasmas considered was compared to that of a circular plasma with the same minor radius, same heat flux and profiles. The ratio of the confinement times, introduced as a factor of merit in shaped plasmas, increases as the elongation $\kappa$ is raised and the triangularity $\delta$ is decreased [2-4]. It is hence of fundamental importance to investigate if the plasma geometry has analogous effects on the transport of moderate-Z impurities and thus to find suitable configurations in which the energy confinement is favoured with respect to that of impurities.

A well proven technique to investigate impurity transport in plasmas consists in injecting very short bursts of impurities in order to separately observe their inward and outward progression in the plasma. In the absence of a source term and apart from effects of sawteeth, the radial transport of impurities is assumed to be governed by the equation:

$$
\frac{\partial n(r)}{\partial t}=\nabla \cdot(D(r) \nabla n(r)-n(r) v(r))
$$

in which the boundary condition $n(a, t)=0$ has to be included and the same transport coefficients $D(r)$ and $v(r)$ are used for all the ionised states. A general and complete solution of Eq.(1) can be written in the form[5]:

$$
n(r, t)=\sum_{k} A_{k} n_{k}(r) \exp \left(-\frac{t}{\tau_{k}}\right)
$$

in which the profile of the eigenfunctions $n_{k}(r)$ and the corresponding time constant $\tau_{k}$ depend on the plasma size and geometry and on the profiles of the transport coefficients. Eq.(2) suggests that, after an initial transient phase, the radial profile of the impurity density, in absence of an external source, tends to assume the shape of the fundamental eigenfunction and to decay with a defined time constant $\tau_{0}$ identified with the impurity confinement time. Moreover, all local or line integrated measurements of impurity radiation decay exponentially with the same time constant $\tau_{0}$. The link of $n_{k}(r), \tau_{k}$ with the plasma geometry and the profiles of transport coefficients $D(r), v(r)$ is not trivial and a connection has to be found by means of a numerical simulation.

The experimental observations of the impurity confinement time on the plasma geometry 
made on TCV are presented in Section 2. Section 3 contains the results of the numerical simulations of impurity transport; the diffusivity and the convective velocity obtained for the same experiments are presented.

\section{Experiment}

The TCV tokamak $\left(R_{0}=0.88 \mathrm{~m}, a<0.25 \mathrm{~m}, B_{T}<1.5 \mathrm{~T}, 1<\kappa<2.8,-0.8<\delta<0.9\right)[1]$ is well suited for plasma geometry studies due to its extreme shaping capabilities, which allow the production of highly elongated plasmas as well as high negative and positive triangularities $[2-4,6]$. The standard laser blow-off technique[7, 8] was used to inject trace (i.e. non perturbing) quantities of silicon into TCV plasmas. Injections were performed during the current plateau, at a rate of 1 per plasma discharge using a single pulse ruby laser.

The 200 channel soft $\mathrm{x}$-ray (SXR) photodiode array with $47 \mu \mathrm{m}$ thick Be filters served as the main diagnostic tool for this study[9]. The spectral sensitivity of the filtered photodiodes ( $>0.5$ for photons in the range $2.5-11 \mathrm{keV}$ ) is well suited for the detection of line emission and bremsstrahlung from highly ionised states of moderate- $Z$ impurities in the core of TCV plasmas. The cameras, whose lines of sight fully cover a single poloidal plane, allow a resolution of 30-40mm after performing a tomographic reconstruction of the local SXR emissivity[9]. The system allows a maximum sampling rate of $80 \mathrm{kHz}$, which is much faster than the typical timescale of the impurity propagation inside a TCV plasma[10].

The global confinement time of impurities in TCV discharges has been determined by fitting an exponential curve to the decaying part of the line integrated SXR signals of both central and peripheral chords $[10,11]$ for which $\rho_{\min }<0.8$. The sawtooth amplitude being of the same order of magnitude as the pulse-shaped signal generated by the impurities, the separation of the two contributions to the SXR signal is necessary. Provided that the background signal and the sawtooth activity are regular, they can easily be separated from the impurity contribution by means of the Generalised Singular Value Decomposition (GSVD) method[12]. A typical result of this technique is shown in Fig.1, where the raw and the "filtered" signals have been superposed. The so filtered signal can easily be characterised by the exponential decay time and by the full width half maximum ( $f w h m)$ which will be used as a matching parameter in transport simulations[10].

For a given injection, all the line integrated SXR signals decay with the same time constant, within the uncertainty of the fitting procedure which is of the order of $1-2 \mathrm{~ms}[10]$. This time constant defines the global impurity lifetime $\tau_{\text {imp }}$ in the plasma. The observation of a 
unique time constant for all line integrated SXR signals is in agreement with Eq.(2) and its consequences reported above.

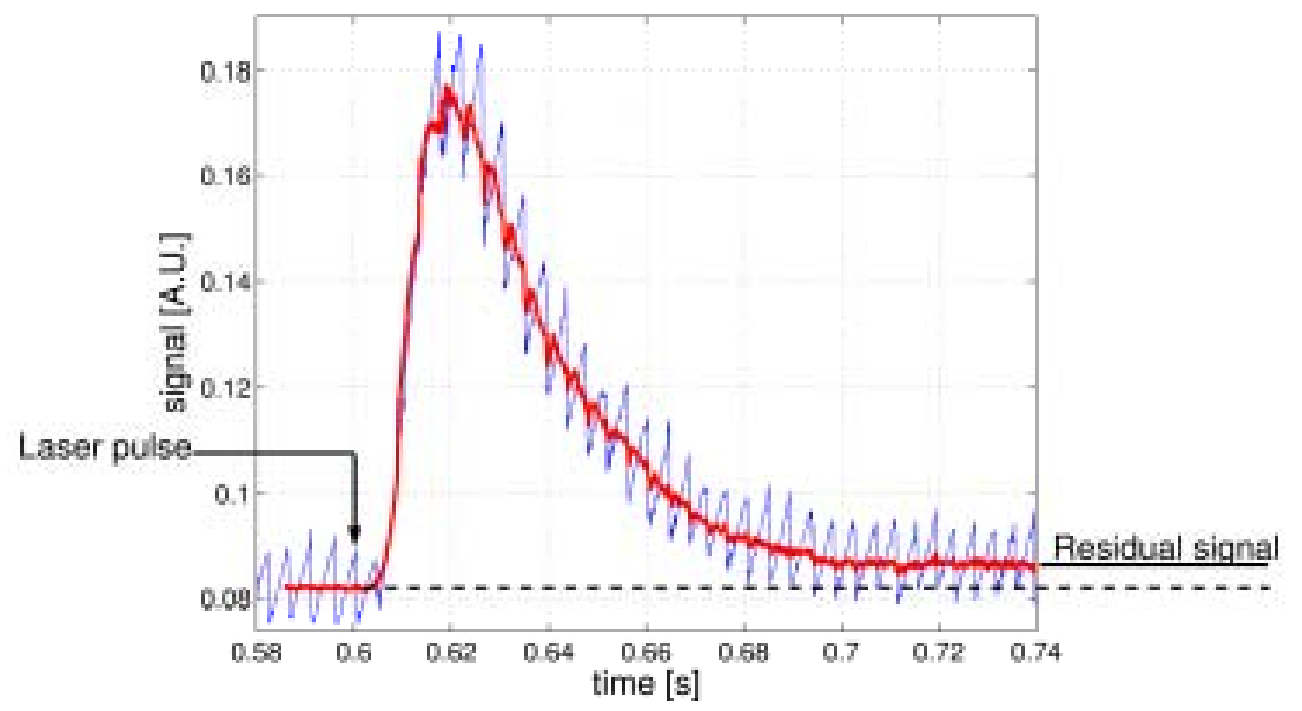

Fig.1 - SXR line integrated signal along a central chord following silicon injection in TCV. The removal of sawteeth, by means of the GSVD technique, allows a more precise evaluation of the time constant of the exponential decay as well as of the full width half maximum of the impurity signal. The residual asymptotic signal is also more defined quantitatively.

Many injection signals end with a residual plateau whose height is typically around 4-6\% of the peak intensity above the background measured before injection. Although silicon is considered to be a non-recycling impurity, this asymptotic residual signal proves that a small amount of silicon is recycled from the vessel walls after the ablation. The recycling of silicon may still be present in subsequent discharges at a constant rate, contributing to the SXR background without affecting further injection experiments.

We present here the results of silicon injection experiments in Ohmic L-mode deuterium TCV discharges[10] in which no MHD activity was present except sawteeth. The central electron temperature being in the range $T_{e 0}=650 \div 850 \mathrm{eV}$ for all discharges, the SXR system was able to detect impurity signals on the chords for which $\rho_{\min }<0.85$. The effects of the plasma shape are presented in a scan of elongation $\kappa$ and a scan of triangularity $\delta$. A dedicated scan of toroidal magnetic field $B_{T}$ is also presented. All the plasmas in which a silicon injection was performed were inner wall limited. For the experiments reported here, no effect of the impurity injection was observed on the electron density $n_{e}$ and the electron temperature $T_{e}$. In particular, no fast inward propagating cold pulses[13] have been observed, suggesting 
that the sudden ionisation of ablated material did not lead to transient changes of the transport properties of the plasma.

\subsection{Scan of elongation}

The scan of elongation in the range $1.6<\kappa_{a}<2.3$ was performed in 2 series of consecutive discharges, consisting of a total of 14 impurity injections. Some configurations were repeated twice in order to control the repeatability of the results; in these cases, the observed impurity confinement time was reproducible within $1-2 \mathrm{~ms}$. Among the other parameters, some were kept constant: the triangularity was $\delta=0.3 \div 0.4$, the line averaged electron density $\bar{n}_{e}=5.6 \div 6.9 \times 10^{19} \mathrm{~m}^{-3}$, the toroidal magnetic field was kept at the value of $B_{T}=0.92 \mathrm{~T}$. The low values of $B_{T}$ and $I_{P}$ reduce the risk of damage in case of disruption at high elongations. The plasma current was varied proportionally to the elongation in the range $I_{P}=350 \div 600 \mathrm{kA}$, such as to keep approximately constant the normalised sawtooth inversion radius $\rho_{i n v}$, the possible dependence of the extension of sawteeth on the impurity confinement was removed. The inversion radius was calculated to be, for all shots of the elongation scan, at $\rho_{i n v} \approx 0.55$, following the scaling laws reported in Ref.[14].

The confinement times of impurities with different plasma elongations are shown in Fig.2(a). The dependence of $\tau_{\text {imp }}$ on elongation is surprisingly different from the case of the
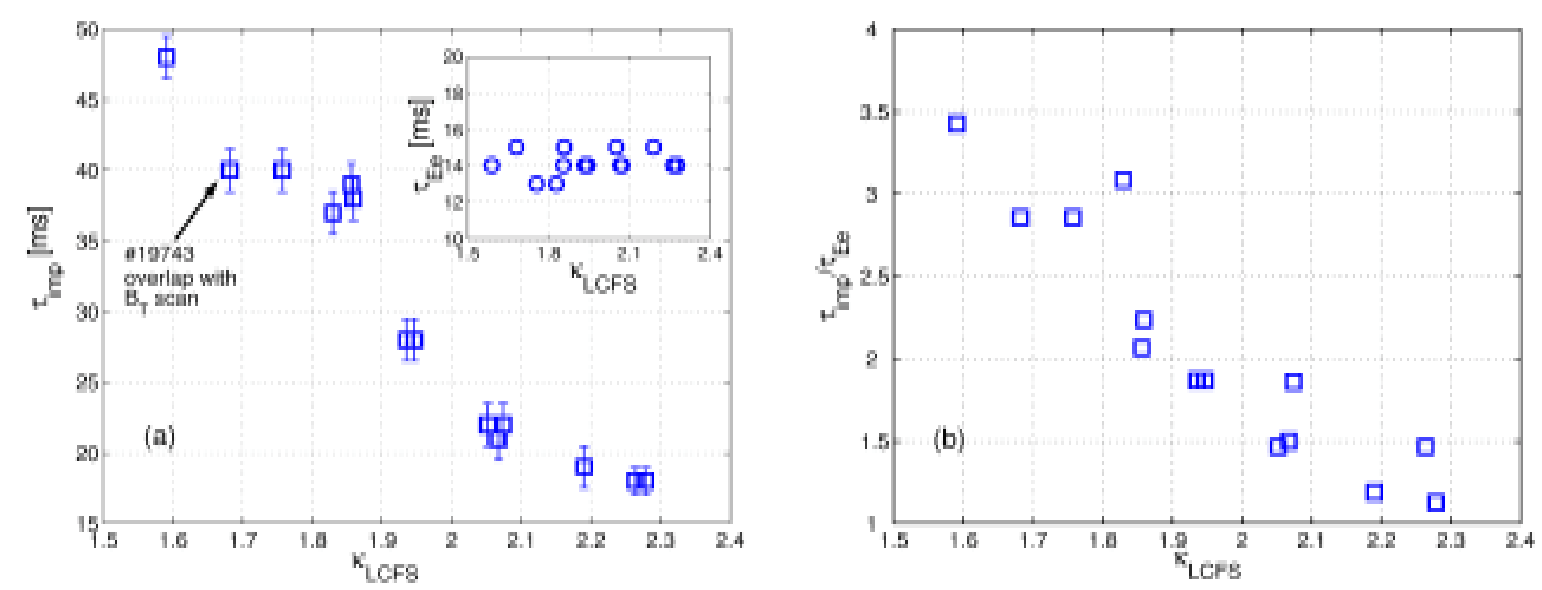

Fig.2 - (a) Confinement times of the injected silicon impurities for different plasma elongations, $1.6<\kappa_{a}<2.3$. In this scan the current was varied accordingly in the range $I_{P}=350 \div 600 \mathrm{kA}$ in order to keep the sawtooth inversion radius constant at $\rho_{i n v}=0.55$. The other parameters were $\delta=0.3 \div 0.4, B_{T}=0.92 \mathrm{~T}$, $\bar{n}_{e}=5.6 \div 6.9 \times 10^{19} \mathrm{~m}^{-3} \cdot \tau_{i m p}$ is uniformly decreasing with elongation. In the inset, the electron energy confinement time for the same discharges is shown, and no correlation with elongation is observed, since $\tau_{E e}$ is constant around $14 \mathrm{~ms}$. (b) The ratio $\tau_{i m p} / \tau_{E e}$ for the same scan. 
electron energy (inferred from Thomson scattering data), decreasing with increasing elongation from relatively high values around $\tau_{\text {imp }}=48 \mathrm{~ms}$ for $\kappa=1.59$ to $\tau_{\text {imp }}=18 \mathrm{~ms}$ for $\kappa=2.28$. If $\tau_{\text {imp }}$ followed the same behaviour as the energy confinement time, it would be expected to moderately increase by about $20 \%$ along the scan.

The plasma elongation appears to be the geometric parameter which exhibits the largest difference in the behaviour of impurities and electron energy. The electron energy confinement time was calculated for the shots of this scan, and the ratio $\tau_{i m p} / \tau_{E e}$ represented in Fig.2(b).

The value of $\tau_{E e}$, which has been calculated according to the definition[6] $\tau_{E e}=(3 / 2) \int n_{e} T_{e} d V / P_{i n}$, is fairly constant during the scan, around $\tau_{E e}=13 \div 15 \mathrm{~ms}$. The lack of dependence on elongation is explained by the simultaneous variation of plasma current, according to the Neo-Alcator-TCV scaling law [4] for which $\tau_{E} \propto \kappa / I_{P}$. As a consequence, the ratio of the confinement times follows the same behaviour as the impurity confinement time alone.

\subsection{Scan of triangularity}

The triangularity scan was performed on 13 non-consecutive discharges. The scan spans the triangularity in the interval $\delta_{a}=-0.16$ to $\delta_{a}=0.64$, while the other plasma parameters are kept as constant as possible. The elongation was $\kappa=1.52 \div 1.63$, the line averaged electron density $\bar{n}_{e}=5.6 \div 6.9 \times 10^{19} \mathrm{~m}^{-3}$, the plasma current $I_{P}=320 \div 380 \mathrm{kA}$ and the toroidal magnetic field $B_{T}=1.47 \mathrm{~T}$. The sawtooth inversion radius was $\rho_{\text {inv }} \approx 0.38$.

The effect of triangularity on the impurity confinement time is shown in Fig.3. For values larger than a threshold around $\delta=0.2$ there is no effect of the plasma triangularity and the impurity confinement time is around $\tau_{i m p}=25 \mathrm{~ms}$. From this scan, as well as from scans of other parameters, shown in the following, the plateau at $\tau_{\text {imp }}=22 \div 25 \mathrm{~ms}$ appears to be a kind of typical value for many different discharge configurations. For $\delta<0.2$, the impurity confinement time increases to much higher values with a similar scaling to that of the electron energy confinement time for the same discharges. Except for the lowest triangularity, the geometry of the magnetic surfaces alone, which causes the increase of $\tau_{E e}$, may account for this behaviour. At the lower end of the scan, it is found that $\tau_{i m p}=127 \pm 3 \mathrm{~ms}$, which corresponds to an increase of a factor of 5 with respect to the almost constant value found for $\delta>0.2$.

A slightly higher recycling was found in the triangularity scan for low values of $\delta$, which could contribute to an increase of the apparent confinement time. The residual line integrated 
signals are $8 \%$ of the peak value at $\delta=0.28$ and $14 \%$ at $\delta=0.06$; the different recycling level will have to be taken into account in STRAHL simulations in order to rule out possible effects.

The values of the electron energy confinement time are shown in the inset of Fig.3. In agreement with the observations reported in Ref.[2,3], $\tau_{E e}$ is increased while reducing the triangularity, and its values range from $\tau_{E e}=13 \mathrm{~ms}$ for $\delta=0.6$ to $\tau_{E e}=26 \mathrm{~ms}$ for $\delta=0.01$. Although the behaviour is slightly different along the scan of triangularity, both the impurity and the electron energy confinement times are doubled at low triangularity and their ratio $\tau_{i m p} / \tau_{E e}$ is almost constant around a factor of 2.

No density dependence in the range $2 \times 10^{19}<\bar{n}_{e}<6 \times 10^{19}$ was found in an independent scan at fixed shape $(\kappa=1.6, \delta=0.26)$.

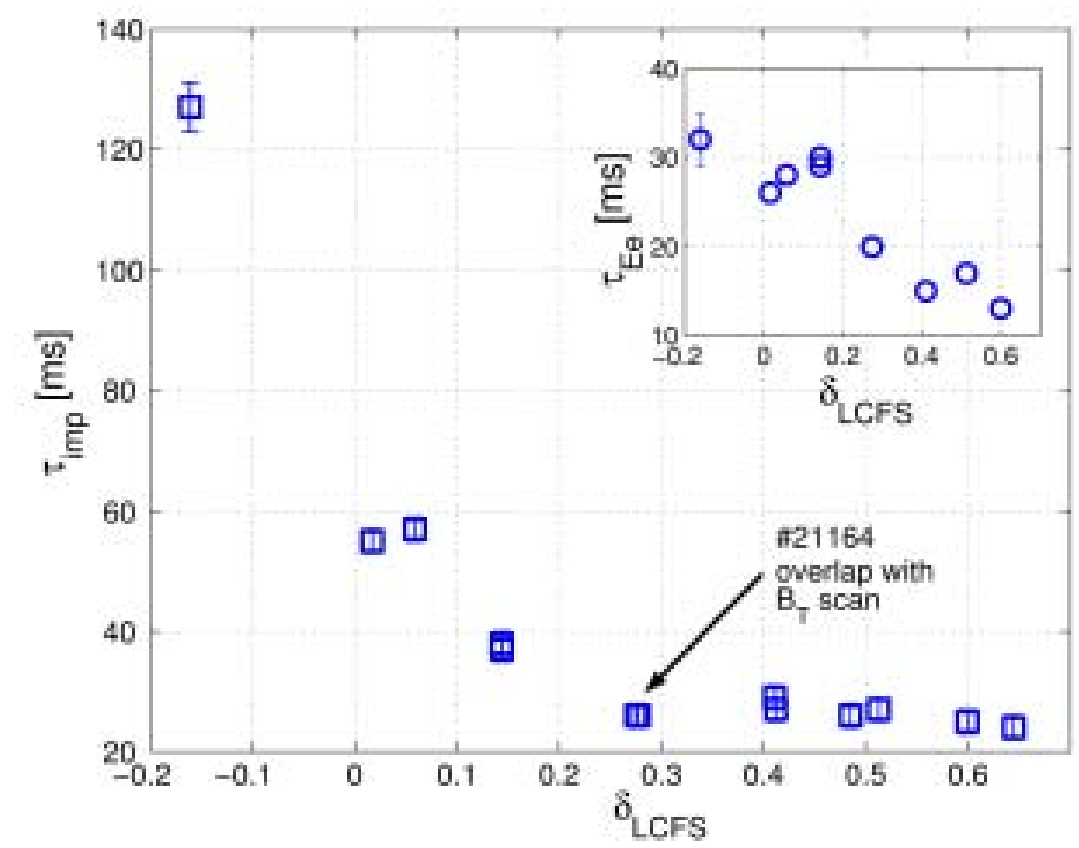

Fig.3 - Confinement time of injected impurities for different plasma triangularities, $-0.16<\delta_{a}<0.60$. The other parameters were $\kappa=1.53 \div 1.62, B_{T}=1.47 \mathrm{~T}, I_{P}=320 \div 380 \mathrm{kA}, \rho_{i n v}=0.38, \bar{n}_{e}=5.6 \div 6.9 \times 10^{19} \mathrm{~m}^{-3} . \mathrm{A}$ threshold appears in the triangularity around $\delta=0.2$, below which the confinement time starts increasing. For selected discharges the electron energy confinement times are shown. Their behaviour is in agreement with the results of Ref.[2, 3]. For the evaluation of the point at $\delta=-0.16$ no direct measurement was available. The extrapolation has been performed with the Neo-Alcator-TCV scaling law[4].

\subsection{Scan of toroidal magnetic field}

It has to be remarked that at the lower end of the elongation scan the plasma geometry, density and current are very similar to those of some discharges included in the triangularity scan, 
but the confinement times are different by a factor of 2 . While the profiles of the electron density and temperature in similar shots are comparable within 10\%, the toroidal magnetic field is the only really different parameter in similar plasmas in the triangularity and the elongation scans. However, the influence of the magnetic field appears to favour the confinement of impurities at lower values of $B_{T}$, in apparent opposition to common sense.

The confirmation of the surprising result for which $\tau_{i m p}$ decreases with increasing $B_{T}$ was obtained from a dedicated scan, which includes at its extremities two discharges already belonging to the triangularity scan (shot $\# 21164, B_{T}=1.47 \mathrm{~T}, \delta=0.28$ ) and to the elongation scan (shot \#19743, $\left.B_{T}=0.93 \mathrm{~T}, \quad \kappa=1.68\right)$. The constant parameters were $\bar{n}_{e}=5.4 \div 6.4 \times 10^{19} \mathrm{~m}^{-3}, \quad \delta=0.28 \div 0.32, \kappa=1.62 \div 1.70$ and $I_{P}=353 \div 356 \mathrm{kA}$ and, as a consequence of the constant plasma current, the sawtooth inversion radius decreased from $\rho_{i n v} \approx 0.55$ to $\rho_{i n v} \approx 0.38$ as the toroidal magnetic field was raised. The result of this scan, shown in Fig.4, is that the increasing magnetic field at constant current produces a regular decrease of the impurity confinement time. If the change in $\tau_{i m p}$ were due to the change in $\rho_{i n v}$, we would expect the opposite trend. At low field, the inversion radius being wider and sawteeth more frequent $\left(v_{S T}=190 \mathrm{~Hz}\right.$ vs $v_{S T}=140 \mathrm{~Hz}$ ), the impurity transport is expected to

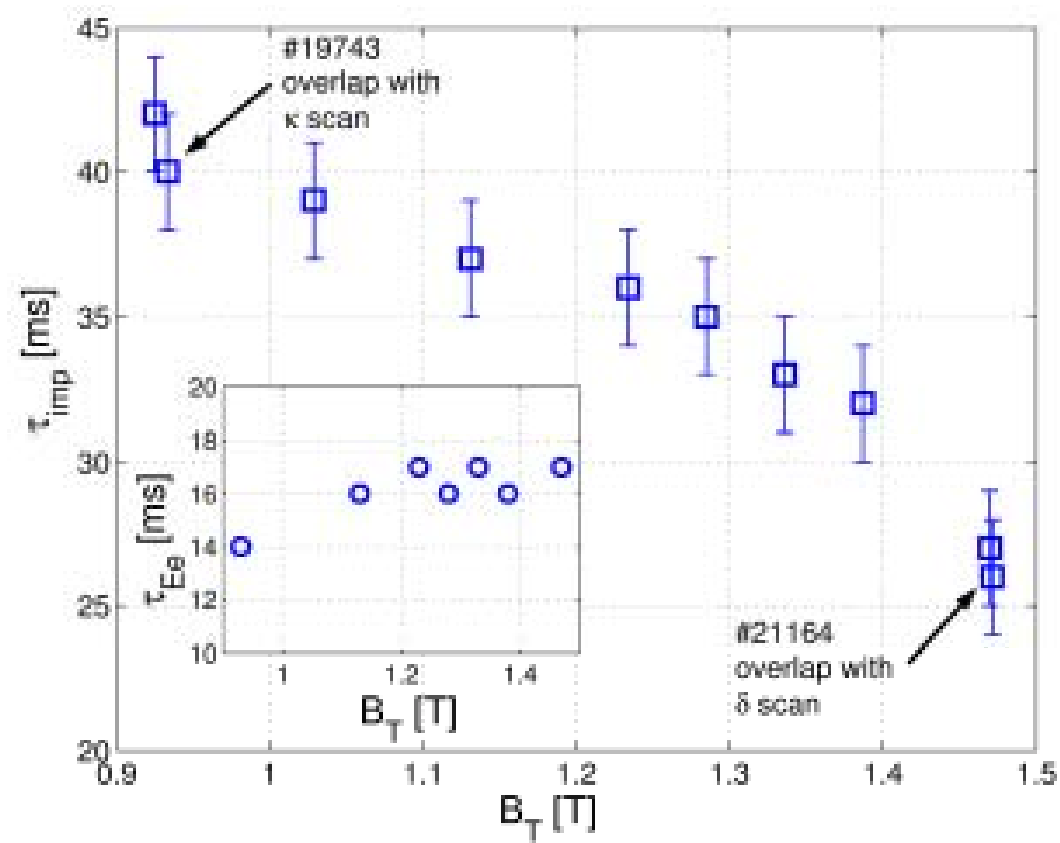

Fig.4 - Dependence of the impurity confinement time on the toroidal magnetic field, $0.92<B_{T}<1.47$, with $\kappa=1.62 \div 1.70, \delta=0.28 \div 0.32 I_{P}=353 \div 356 \mathrm{kA}, \rho_{i n v}=0.38 \div 0.55, \bar{n}_{e}=5.4 \div 6.4 \times 10^{19} \mathrm{~m}^{-3}$. In the inset, the electron energy confinement time for selected shots in the same scan. 
be larger during sawtooth collapses and the confinement time to be lower.

For the same series of discharges, the electron energy confinement time has been calculated and is fairly independent on the toroidal magnetic field for the values investigated in this scan, as it ranges from $\tau_{E e}=14 \mathrm{~ms}$ for $B_{T}=0.92 \mathrm{~T}$ to $\tau_{E e}=17 \mathrm{~ms}$ for $B_{T}=1.47 \mathrm{~T}$ (inset of Fig.4).

\section{Transport simulations}

A technique[13,15-18] which is widely used to give quantitative estimates of the transport parameters for impurities in plasmas consists in calculating the evolution of the concentration of the injected impurities and derive the electromagnetic radiation which would be measured on the basis of the assumed transport parameters. The simulated signals are compared with the experimental ones in order to find the bestfitting set. As a common feature, the plasma background during simulation is assumed not to be perturbed by small amounts of injected impurities.

The STRAHL code[19] has been used on TCV for the study of the impurity injections shown in Section 2. The bulk plasma parameters, namely the electron density and temperature profiles, the plasma geometry, the sawtooth inversion radius and frequency, are taken from the experimental data. The profiles of the diffusivity and the convective velocity are set as free input parameters and their values were determined by simulating the evolving line integrated SXR signals. We chose to reproduce, by means of the code, the time evolution of the line integrated SXR signals from many central and mid-radius chords $\left(\rho_{\min }<0.6\right)$, instead of the tomographically inverted SXR profiles, as the determination of the transport parameters seemed to be more accurate. A consistency check can be made a posteriori by comparing the resulting simulated profile with an experimental, tomographically inverted profile, as well as by comparing the experimental and the simulated line integrated SXR signals for a number of central and peripheral chords.

The neoclassical predictions, which are presented in the following for comparison with the experimental data, have been obtained by means of the NEOART package[16, 20] included in STRAHL. In the experiments presented in this work, the silicon in TCV Ohmic plasmas lies in the high collisionality Pfirsch-Schlüter regime (characterised by $v^{*}>1$ ) over all the plasma volume. However, the classical and banana-plateau contributions were taken into account for the calculation of the neoclassical values of $D$ and $v$. The transport coefficients $D_{\text {neo }}$ and $v_{\text {neo }}$ presented in the following are actually the sum of all three contributions. 


\subsection{Experimental values of $D$ and $v$}

The following results have been obtained from simulations in which the radial profile of diffusivity was parametrised with a parabola, $D(\rho)=D_{0}+\left(D_{\text {edge }}-D_{0}\right) \rho^{2}$ while the profile of the convective velocity was assumed to be linear, $v(\rho)=v_{\text {edge }} \rho$. Different profiles were attempted, such as an exponential dependence for the diffusivity or a parabolic one for the velocity. Although it was not possible to determine with precision the profiles of $D$ and $v$, the best agreements were found with the above parametrisations. In particular, as observed in many other experiments, the core diffusivity $D_{0}$ is found to be much lower than the edge one, $D_{\text {edge }}$.

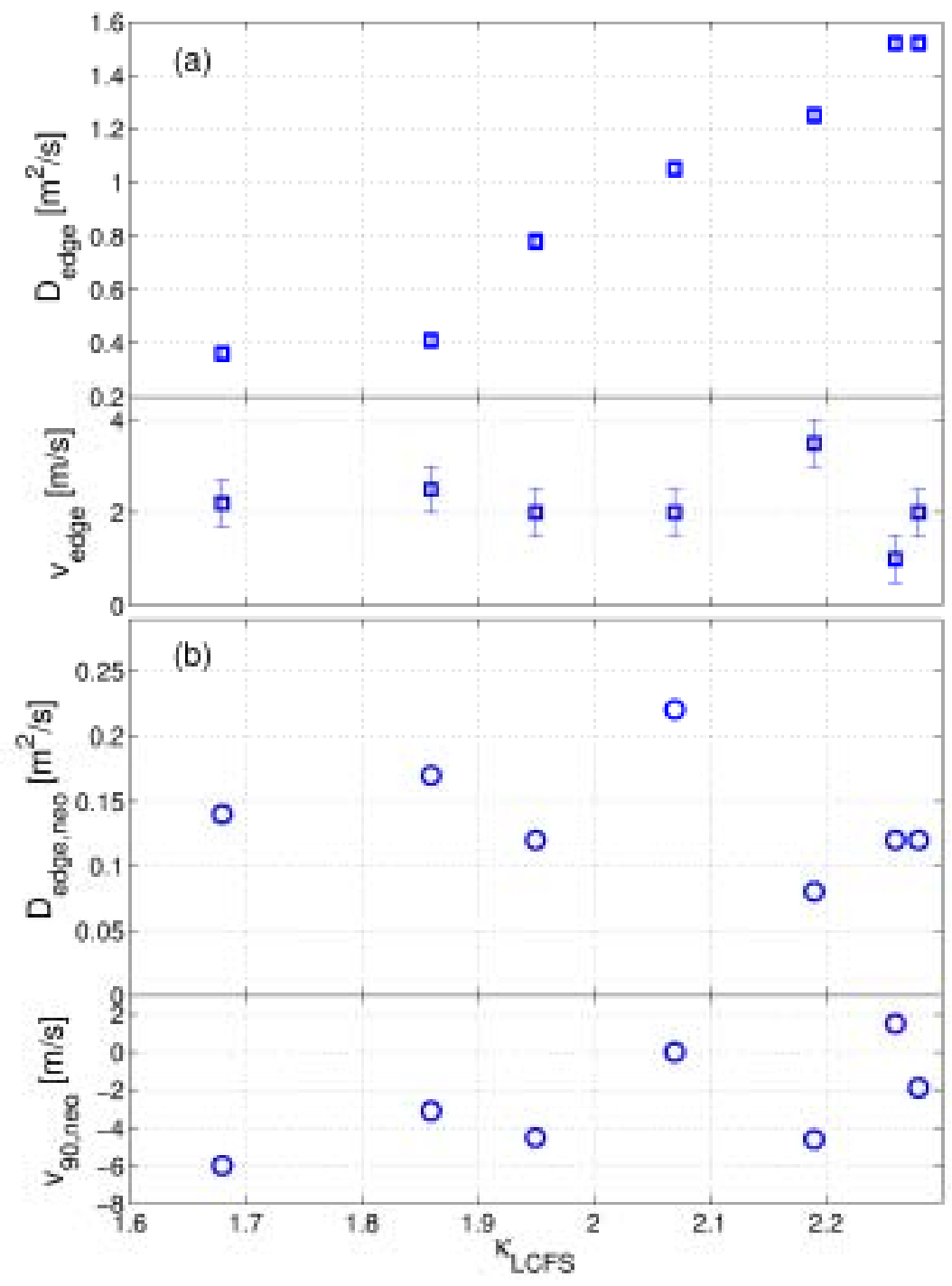

Fig.5 - (a) Bestfitting parameter couples $\left(D_{\text {edge }}, \mathrm{v}_{\text {edge }}\right)$ used to reproduce the time evolution of the line integrated SXR signals of selected shots in the scan of elongation shown in Fig.2. (b) The neoclassical values of the diffusivity at the LCFS and the convective velocity at $\rho=0.9$ as calculated by NEOART for the same discharges. 
The transport parameters at the plasma edge were easily determined by requiring that in the simulated signals the values of the time constant of the exponential relaxation and the signal fwhm matched those found in the experimental signals. In the plasma core, the diffusion coefficient can be evaluated in the early phase of the impurity transport in a plasma, when the signal increases between two consecutive sawteeth, as the central impurity profile is hollow and steep. During the outward progression of the impurities, the central profile has been almost flattened out by many sawteeth collapses and the outflux of impurities is not sensitive to the value of the central diffusivity. Unfortunately, $D_{0}$ is largely undetermined for most cases, as it is strongly affected by the uncertainty of the relative timing between the instant at which the impurities start entering the plasma and the first sawtooth crash. An uncertainty of $0.5 \mathrm{~ms}$ can lead to an indetermination of the central diffusivity in the range $D_{0}<0.3 \mathrm{~m}^{2} / \mathrm{s}$. In some cases, however, the SXR line integrated signals were clear enough to determine such relative timing with precision and a value of the central diffusivity was obtained. The values of $D_{0}$ reported in Table 1 have been determined to a precision of $\pm 0.01 \mathrm{~m}^{2} / \mathrm{s}$ by simulating the correct increase of the signal between the first and the second sawtooth following injection. The comparison with the neoclassical predictions shows that the bestfitting central value of the diffusivity is in agreement, within the uncertainty of the measurement, with the neoclassical values[16].

\begin{tabular}{|c|c|c|c|l|}
\hline Shot \# & $\begin{array}{c}\tau_{\text {imp }} \\
{[\mathbf{m s}]}\end{array}$ & $\begin{array}{c}\boldsymbol{D}_{\mathbf{0}} \\
{\left[\mathbf{m}^{2} / \mathbf{s}\right]}\end{array}$ & $\begin{array}{c}\boldsymbol{D}_{\mathbf{0}} \text { neo } \\
{\left[\mathbf{m}^{\mathbf{2}} / \mathbf{s}\right]}\end{array}$ & \multicolumn{1}{|c|}{ Remarks } \\
\hline \hline 21164 & 27 & 0.03 & 0.04 & Top end in $\mathrm{B}_{\mathrm{T}}$ scan at $\mathrm{B}_{\mathrm{T}}=1.47 \mathrm{~T} ; \delta$ scan at $\delta=0.28$ \\
\hline 19742 & 38 & 0.07 & 0.08 & K scan at $\kappa=1.86$ \\
\hline 19782 & 57 & 0.08 & 0.07 & $\delta$ scan at $\delta=0.06$ \\
\hline
\end{tabular}

Table 1: Central impurity diffusivity for some selected discharges

The results of the simulations for the scan of elongation are shown in Fig.5(a). The entire dependence of the impurity confinement time on the elongation is due to a change in the diffusivity, while the convective velocity is fairly constant around the value $v_{\text {edge }} \approx 2 \mathrm{~m} / \mathrm{s}$ and always outwardly directed. The increase of diffusivity with increasing elongation accounts for the reduction of confinement time, shown in Fig.2(a), and for the increase in the size of the plasma. The calculated value of diffusivity at low elongation, $D_{\text {edge }} \approx 0.36 \mathrm{~m}^{2} / \mathrm{s}$ for $\kappa_{a}=1.68$, is a typical constant value which is found in the scans of toroidal magnetic field and triangularity. The neoclassical edge values of the diffusivity and convective velocity, reported in 
Fig.5(b) are lower than the bestfitting ones of a factor of 2.5 at low elongation up to a factor of 10 at the top end of the scan. The neoclassical diffusivity appears not to depend on elongation, while the inward edge velocity is reduced with increasing $\kappa$. In a case at $\kappa=2.26$ the neoclassical velocity is found to be outwardly directed. In this scan, like in all other cases, the convective velocity -indicated as $\mathrm{v}_{90, \text { neo- }^{-}}$is evaluated at $\rho=0.9$, as the edge value appears to be strongly affected by the uncertainty in the experimental determination of the edge values of a number of plasma parameters.

The reduction of $\tau_{\text {imp }}$ with increasing toroidal magnetic field is fully due to an increase in

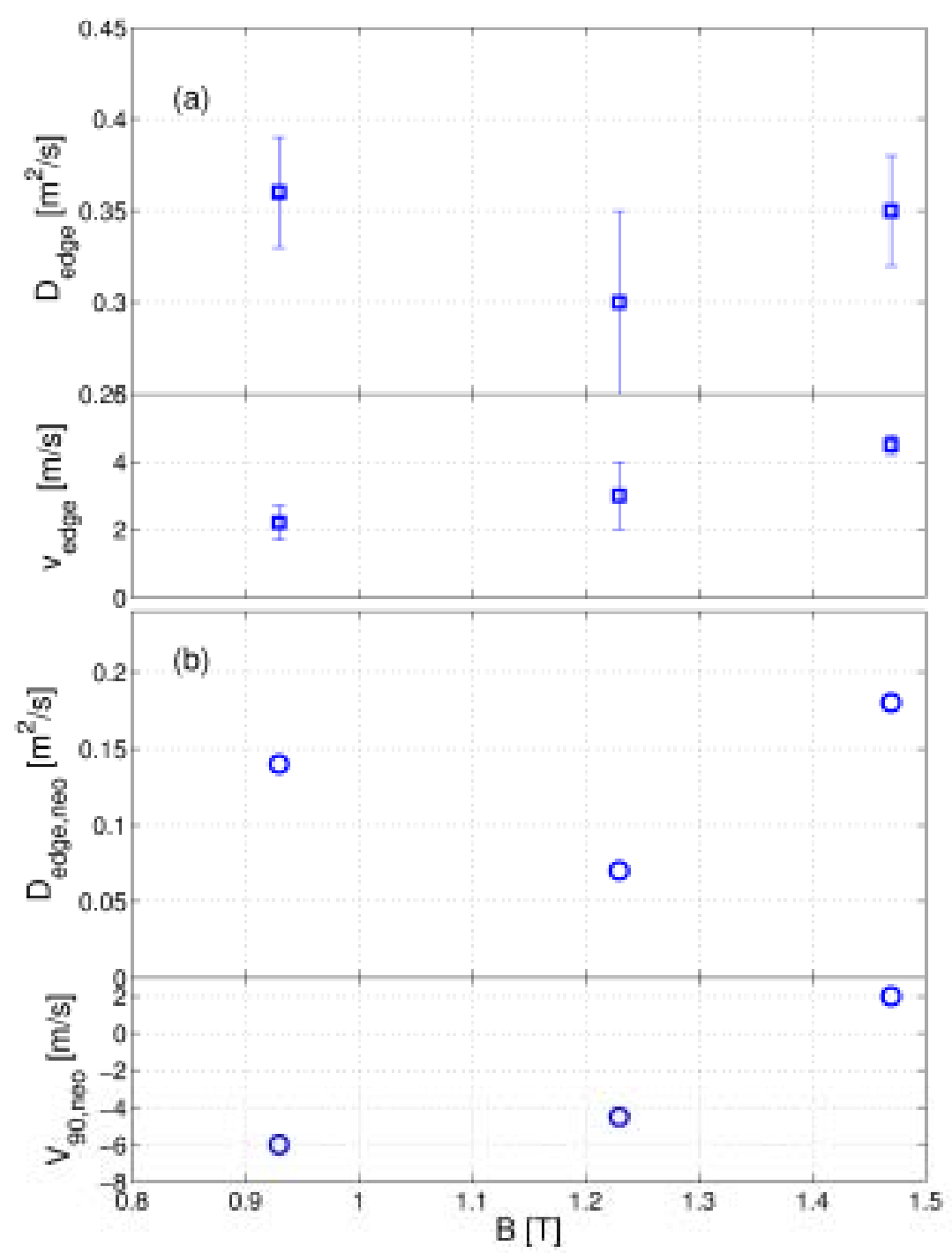

Fig.6 - (a) Bestfitting parameters $\left(D_{\text {edge}}, \mathrm{v}_{\text {edge}}\right)$ as a function of the toroidal magnetic field. The point at $\mathrm{B}_{\mathrm{T}}=0.93 \mathrm{~T}$ belongs also to the elongation scan at $\kappa=1.68$, while the point at $\mathrm{B}_{\mathrm{T}}=1.47 \mathrm{~T}$ is part of the triangularity scan at $\delta=0.28$. (b) The neoclassical values of the diffusivity at the LCFS and the convective velocity at $\rho=0.9$ as calculated by NEOART for the same discharges. 
the radial convective velocity whose value goes from $v_{\text {edge }} \approx 2 \mathrm{~m} / \mathrm{s}$ to $v_{\text {edge }} \approx 4.5 \mathrm{~m} / \mathrm{s}$ and is directed outward. In Fig.6 the results of the simulation on selected injections are shown. Like in the elongation scan, the neoclassical edge diffusivity lies below the value of $0.2 \mathrm{~m}^{2} / \mathrm{s}$ and it is lower than the bestfitting values of at least a factor of 2 . The neoclassical velocity changes with the magnetic field and again in one case at $B_{T}=1.47 \mathrm{~T}$ it is positive. The dependence of the convective velocity on the triangularity is evident in Fig.7. In this scan, performed in the range $\delta=-0.16 \div 0.6$ the impurity confinement time was fairly constant around $25 \mathrm{~ms}$ for $\delta>0.2$ and increasing for lower values to negative triangularities.

The bestfitting transport parameters show that the edge diffusivity is constant or just

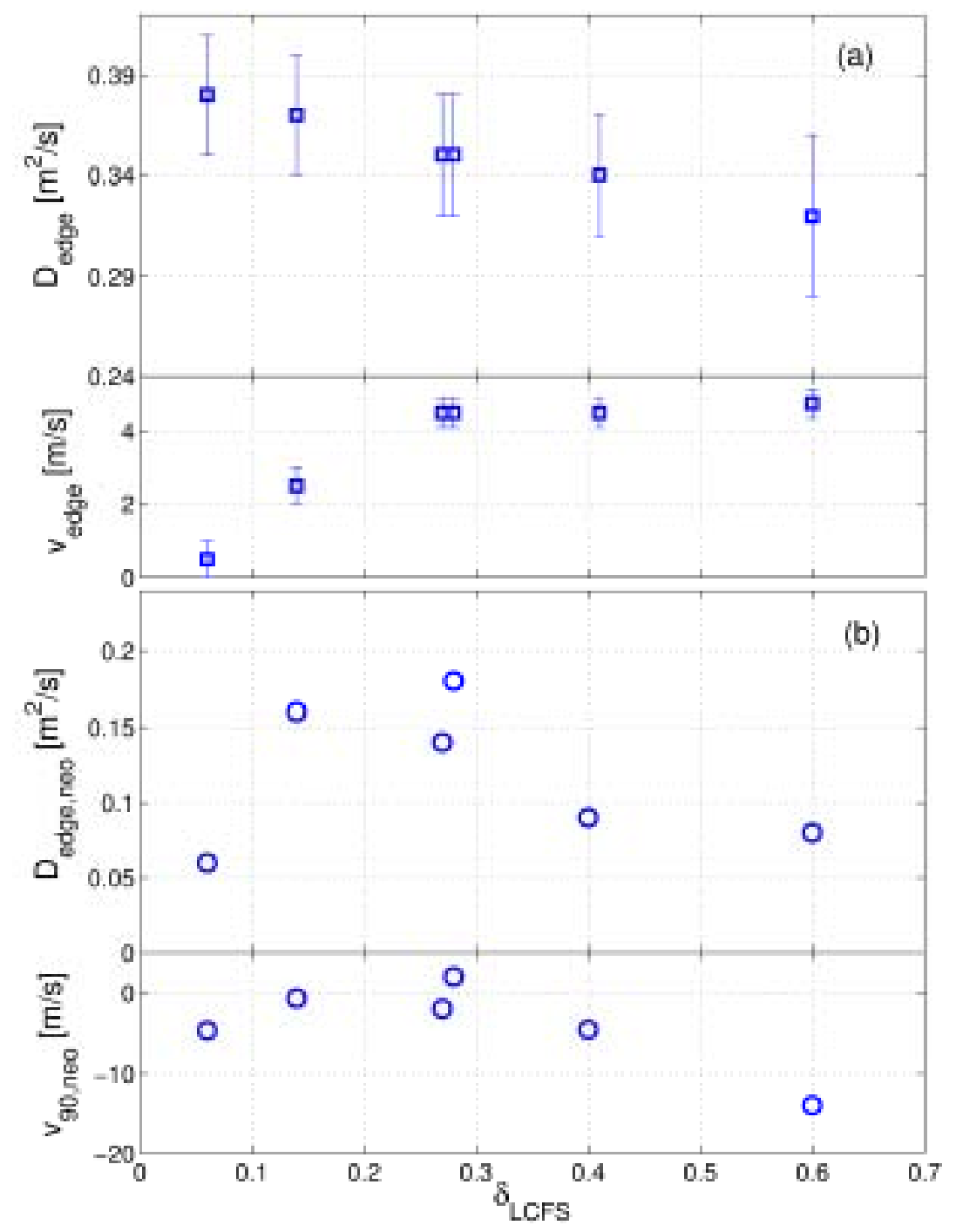

Fig.7 - (a) Dependence of the edge diffusivity and the convective velocity on the plasma triangularity. (b) The neoclassical values of the diffusivity at the LCFS and the convective velocity at $\rho=0.9$ as calculated by NEOART for the same discharges. 
slightly decreasing, while the confinement time behaviour represented in Fig.3 is correlated to a change in the edge convective velocity. Its values lie around $v_{\text {edge }} \approx 4.5 \mathrm{~m} / \mathrm{s}$ until the threshold at $\delta=0.2$ then a decrease is observed in correspondence of the increase of the impurity lifetime. At $\delta=0.06$ the edge velocity has fallen to around 0 . Unfortunately it was not possible to simulate the signal at $\delta=-0.16$ due to the lack of a number of experimental data.

The neoclassical values in the scan of triangularity are similar to those found previously. Both the diffusivity and the convective velocity are not clearly correlated to the triangularity; the values of $D_{\text {edge }}$ lie around $0.1 \div 0.15 \mathrm{~m}^{2} / \mathrm{s}$, a factor of 2 at least below the bestfitting values, and the velocity at $\rho=0.9$ is slightly negative, except in one case.

\subsection{Testing the $v>0$ result}

As a general result in all of the simulations presented here, the bestfitting values of the convective velocity were always found positive, i.e. outwardly directed. This result, which has never been observed in other experiments, can be tested by comparing the profiles of the SXR local emissivity obtained with tomographic inversion of actual measurements with the SXR profile which is obtained from the fundamental eigenfunctions which are solution of Eq.(1).

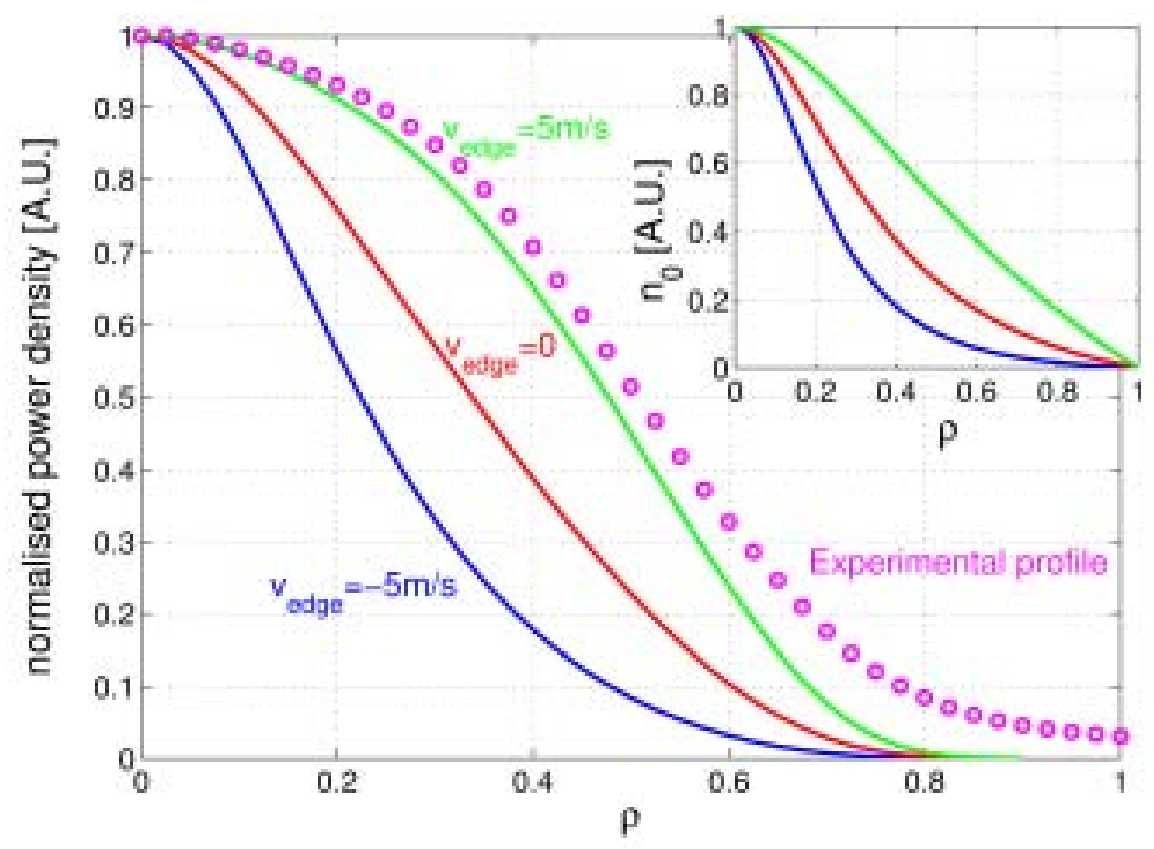

Fig.8 - SXR profiles corresponding to the fundamental eigenfunction of Eq.(2), for different assumption on the edge velocity, compared to the tomographically inverted profile of the impurity SXR emission of shot \#21164 $\left(\mathrm{B}_{\mathrm{T}}=1.47 \mathrm{~T}, \quad \delta=0.28\right)$ during the exponentially decaying phase. The bestfitting diffusivity is $D(\rho)=0.03+0.32 \rho^{2} \mathrm{~m}^{2} / \mathrm{s}$. In the inset, the fundamental impurity distributions are shown. The excessively high experimental profile at the edge may result from the regularisation procedure in the tomographic inversion. 
In Fig. 8 this comparison is shown for the injection in discharge \#21164, which belongs to both the triangularity and the magnetic field scan. The diffusion coefficient was kept constant in the numerical calculation of the solution of Eq.(1), equal to the bestfitting one, $D(\rho)=0.03+0.32 \rho^{2}$, while three different velocity profiles were used. The corresponding fundamental eigenfunctions, shown in the inset of Fig.8, have been used to calculate the profile of emissivity in the SXR spectral range. The experimental profiles of the electron density and temperature were taken, while we simply used the corona approximation for calculating the radiation profiles. This approximation, which neglects the effects of cross-field transport on the ionisation equilibrium, is justified after many independent comparisons showed that the difference with the experimental SXR profiles and the corona predictions never exceeded $5 \%[10]$.

The comparison was made with a tomographically inverted profile of the impurity density obtained when the higher modes have disappeared and only the fundamental distribution is left, typically 30ms after the peak of the line integrated SXR signals. As shown in Fig.8, the profile obtained with $v_{\text {edge }}=5 \mathrm{~m} / \mathrm{s}$ is very close to the experimental one, which is consistent with the bestfitting value $v_{\text {edge }}=4.5 \mathrm{~m} / \mathrm{s}$.

\section{Conclusions}

Small quantities of silicon were injected into TCV by laser blow-off to study impurity transport in shaped L-mode Ohmic discharges. The results show that the plasma geometry has important effects on the impurity confinement time and consequently on the impurity transport coefficients. The most surprising observations were obtained from the scan of elongation, in which the increase of $\kappa$ leads to a marked reduction of the impurity lifetime. For the same shots, the electron energy confinement time is found constant and hence a favourable ratio $\tau_{\text {imp }} / \tau_{E e}$ is obtained. Similarly, the increase of the toroidal magnetic field leads to lower values of the impurity lifetime and higher ratios $\tau_{E e} / \tau_{i m p}$, while in the triangularity scan both $\tau_{i m p}$ and $\tau_{E e}$ increase for low and negative values of $\delta$.

The bestfitting values of the transport parameters $D_{0}, D_{\text {edge }}$ and $v_{\text {edge }}$ were evaluated for selected injections by simulation, in order to reproduce the experimental fwhm and exponential decay of the measured line integrated soft x-ray signals. The behaviour of the impurity lifetime in the elongation scan is correlated to a regular increase of the edge diffusivity and constant outward convective velocity, while the longer values of $\tau_{i m p}$ at low triangularity and 
toroidal magnetic field have been explained with lower convective velocity and fairly constant values of $D_{\text {edge }}$.

In none of the cases the transport coefficients at the plasma edge match those provided by the neoclassical theory. In all the injections presented in this work, the bestfitting edge diffusivity is larger than the theoretical value of at least a factor of 3, up to a factor of 10 for high elongations. More evident is the discrepancy in the edge convective velocity, which is always found to be positive, i.e. outwardly directed, while the neoclassical theory predicts negative values.

The central diffusion coefficient, which was estimated for a small set of injections, is found to be much closer to the neoclassical predictions. It can therefore be assumed that in the plasma centre the impurity transport can be described by the neoclassical theory between consecutive sawtooth collapses.

The diffusivity in the peripheral plasma must be due to an anomalous process, which is not trivially related to the one responsible for energy transport. The lack of understanding of the impurity transport makes it difficult to extrapolate the favourable behaviour of $\tau_{E e} / \tau_{i m p}$ at high elongation to reactor conditions, where collisionalities are 2 orders of magnitude lower.

\section{Acknowledgements}

This work was partly supported by the Swiss National Science Foundation.

\section{References}

[1] F.Hofmann et al., Plasma Phys. Contr. Fusion 36 (1994), B277

[2] J.M.Moret et al., Phys. Rev. Lett. 79 (1997), 2057

[3] H.Weisen et al., Nucl. Fusion 37 (1997), 1741

[4] H.Weisen et al., Plasma Phys. Control. Fusion 39 (1997), B135

[5] F.H.Seguin et al., Phys. Rev. Lett. 51 (1983), 455

[6] F.Hofmann et al., Plasma Phys. Control. Fusion 43 (2001), A161

[7] J.F.Friichtenicht, Rev. Sci. Instr. 45 (1974), 51

[8] E.S.Marmar et al., Rev. Sci. Instr. 46 (1975), 1149

[9] M.Anton et al., Plasma Phys. Control. Fusion 38 (1996), 1849

[10] E.Scavino, PhD Thesis No. 2677, Ecole Polytechnique Fédérale de Lausanne, Switzerland (2002); CRPP-EPFL report LRP 758/03 (2003)

[11] E.S.Marmar et al., Nucl. Fusion 22 (1982), 1567 
[12] T.Dudok de Wit et al., Phys. Plasmas 5 (1998), 1360

[13] M.Mattioli et al., Nucl. Fusion 38 (1998), 162

[14] H.Weisen et al., Nucl. Fusion 42 (2002), 136

[15] M.Mattioli et al., Nucl. Fusion 38 (1998), 189

[16] R.Dux et al., Nucl. Fusion 39 (1999), 1509

[17] D.Pasini et al., Nucl. Fusion 30 (1990), 2049

[18] K.W.Gentle et al., Plasma Phys. Control. Fusion 26 (1984), 1407

[19] K.Behringer, Jet report JET-R(87) 08 (1987)

[20] R.Dux, STRAHL User Manual (2000) 